\title{
Norois
}

Environnement, aménagement, société

\section{Géohistoire d'une trajectoire paysagère dans la plaine du Rhône valaisan. Analyse du secteur entre Riddes et Martigny (1840-1965)}

Geohistory of landscape trajectory of the Rhone River plain in Valais. Sector analysis between Martigny and Riddes (1840-1965)

\section{Dominique Baud et Emmanuel Reynard}

\section{OpenEdition \\ Journals}

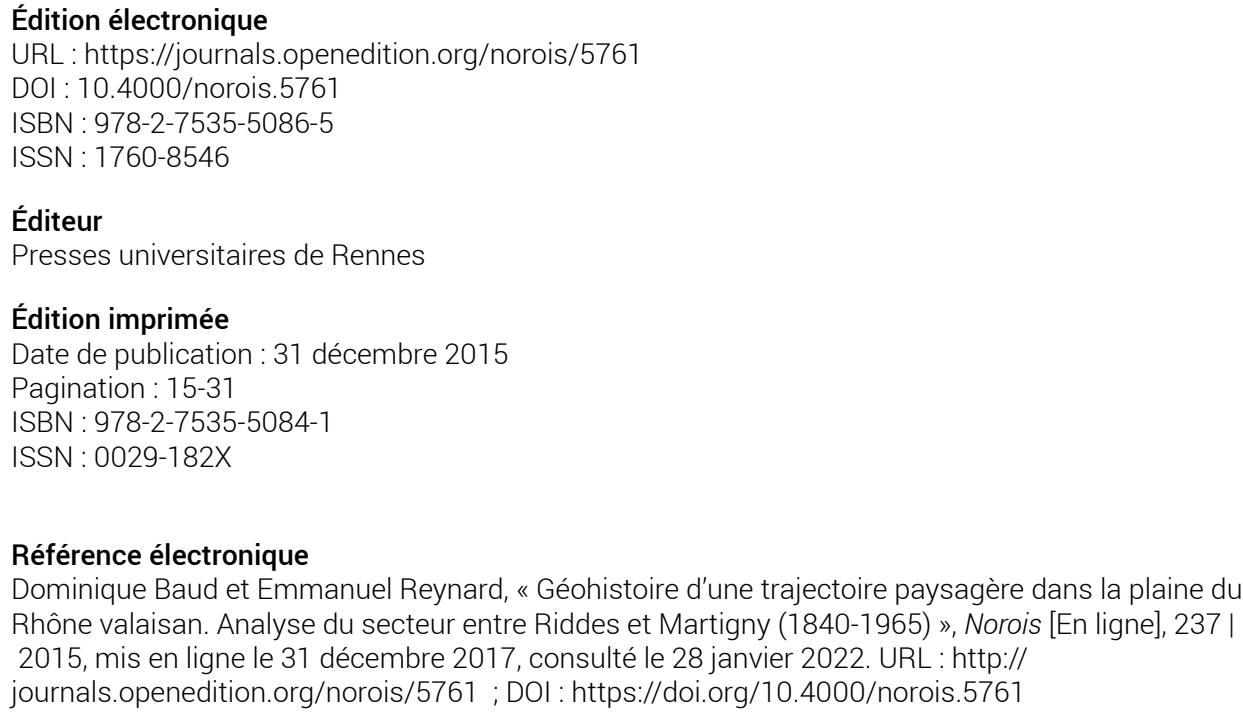




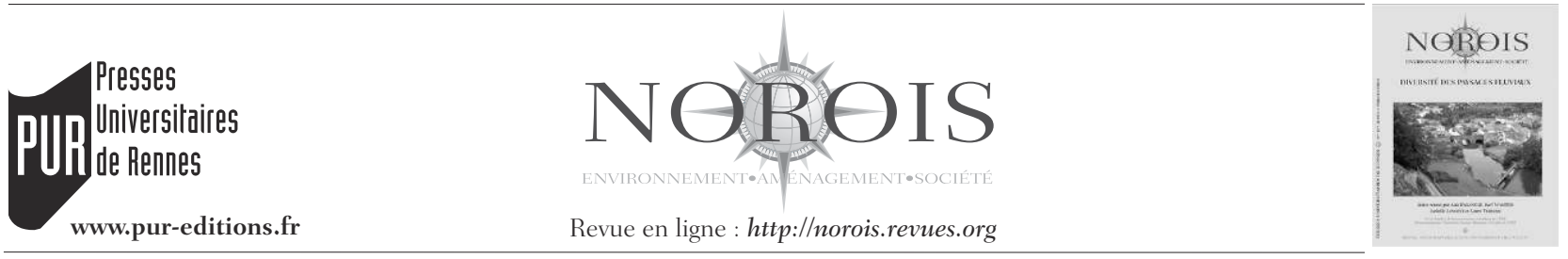

\title{
Géohistoire d'une trajectoire paysagère dans la plaine du Rhône valaisan Analyse du secteur entre Riddes et Martigny (1840-1965)
}

\author{
Geohistory of Landscape Trajectory of the Rhone River Plain in Valais \\ Analysis of the River Section between Riddes and Martigny (1840-1965)
}

\author{
Dominique Baud ${ }^{* a}$, Emmanuel Reynard ${ }^{\mathrm{b}}$
}

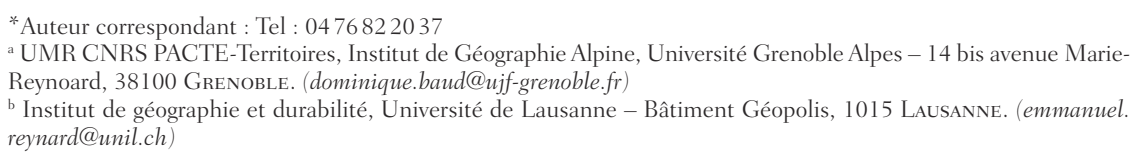

Résumé : Le Rhône à l'amont du lac Léman, dans sa partie suisse, a vécu d'importants travaux d'aménagement (endiguement, rectification du cours) dès 1863. Cet aménagement a été à l'origine d'une transformation radicale du paysage fluvial, qui a vu progressivement les parcelles de cultures intensives (arboriculture, cultures maraîchères) remplacer progressivement les anciennes zones humides et les forêts alluviales. Cette transition paysagère a toutefois duré une centaine d'années et n'a été ni linéaire dans le temps ni homogène dans l'espace. L'approche géohistorique, qui exploite à la fois les sources iconographiques et cartographiques et les archives textuelles, permet non seulement de reconstituer de manière fine la trajectoire paysagère mais aussi d'analyser les raisons des transformations et les dynamiques sociales et politiques à l'origine des transformations paysagères.

\begin{abstract}
The Rhone River upstream of Lake Geneva (Swiss part) has been concerned with large development works (embankments, course rectification) since 1863. These works have provoked radical changes in the alluvial landscape. Progressively, intensive agriculture (arboriculture, vegetable crops) has replaced former wetlands and allwvial forests. The duration of this landscape transition was more than one century and the transition was neither linear in time nor homogeneous in space. The geohistorical approach, which analyses iconographic and cartographic sources and textual archives, allows us not only to reconstruct with precision the landscape trajectories but also to analyse the factors that influenced changes and the social and political dynamics that were at the origin of landscape changes.
\end{abstract}

Mots clés : géohistoire - paysages fluviaux - aménagements - Rhône - transition paysagère

Keywords: geohistory - fluvial landscapes - river training - Rhone River - landscape transition

\section{INTRODUCTION}

Comme de nombreux grands fleuves européens, le Rhône a été au cœur d'importants travaux d'aménagement dans le but de prémunir les sociétés rive- raines des débordements du cours d'eau au cours des deux derniers siècles. En Suisse, dans sa section amont entre Sion et Martigny, des opérations de grande envergure ont été réalisées sur le fleuve afin de fixer un tracé instable évoluant au fil des 
crues. Ces phases d'aménagement - appelées respectivement première (1863-1894) et deuxième (1930-1960) correction du Rhône - ont eu pour corollaire de profondes transformations du paysage fluvial. Dans cet article, nous utiliserons le concept de paysage dans son acception large le définissant comme le produit visible de l'organisation des territoires, résultant de l'action combinée de facteurs naturels et humains (Périgord et al., 2012).

D'une plaine marquée par une forte emprise du cours d'eau, caractérisée par de larges bandes de tressage à la fin du XvIII ${ }^{\mathrm{e}}$ siècle, par la présence de nombreuses zones humides (forêts alluviales, marais, mares temporaires) et par une utilisation agricole extensive (pâture), on passe en un siècle (1860-1960) à un paysage fortement anthropisé, marqué par une mise en valeur agricole intensive (vergers, cultures maraîchères, cultures spéciales) de la plaine alluviale, par le développement d'infrastructures de transport multiples (chemin de fer, routes, autoroute) et, de plus en plus, par une extension des surfaces bâties (Stäuble et Reynard, 2005 ; Zanini et al., 2006; Baud et al., 2015). En cent ans, on glisse progressivement d'un paysage en mouvement partiellement redéfini à chaque crue à un paysage géométrique, guidé par les différents aménagements (digues, canaux de drainage, chemins de desserte).

Nous proposons dans ce texte de définir par l'expression «transition paysagère » cette phase d'évolution qui part d'un état caractérisé par des processus anthropiques dominants dans le cadre d'un système spatial et qui aboutit à d'autres processus générant une organisation spatiale différente (Clément, 1999). Aux deux extrémités de cette transition paysagère, on trouvera " une situation de concordance entre l'humanisation et la spatialisation » (Clément, 1999).

Le paysage actuel conserve les traces héritées du passé qui sont autant de témoins permettant de reconstituer, du moins partiellement, les anciens paysages du Rhône. Afin d'identifier et de comprendre les trajectoires des paysages fluviaux, nous adoptons une approche géohistorique qui vise à restituer à la fois la dynamique et la structuration des territoires ou des paysages sur le temps long à partir de l'exploitation de sources iconographiques et textuelles (Jacob-Rousseau, 2009).
Ce travail de reconstitution des dynamiques paysagères s'inscrit dans le cadre du projet Sources $d u$ Rhône, - mené conjointement par des historiens et des géographes, sous l'égide des Archives de l'État du Valais, dont le but est de valoriser les sources archivistiques diverses sur le Rhône -, et des activités de l'association Mémoires du Rhône, dont l'objectif est de susciter et d'animer des recherches interdisciplinaires sur l'histoire du Rhône suisse et de ses relations avec les riverains (Reynard et al., 2009; Reynard et al., 2015).

Dans cet article, nous nous focalisons sur la région comprise entre Riddes et Martigny (figure 1), qui a été choisie comme zone d'étude du projet Sources du Rhône autant pour l'abondance des sources archivistiques que pour les transformations radicales que la région a connues au cours des derniers siècles, et analysons les trajectoires paysagères entre 1840 et 1965. Le laps de temps étudié correspond à cette période de bouleversement du paysage durant laquelle la plaine passe d'un milieu naturel alluvial à un territoire fortement contraint par les activités humaines, où le paysage est en transition entre deux formes d'organisation bien distinctes. Nous montrerons toutefois que le rythme des transformations n'a pas été linéaire et qu'il existe un profond décalage temporel entre l'aménagement du fleuve et les transformations territoriales (et donc paysagères) de la plaine alluviale. Nous essaierons de dégager les raisons de ce décalage et d'identifier et hiérarchiser les facteurs explicatifs des changements observés. En conclusion, nous discuterons de la relation entre ces changements paysagers et les enjeux de la troisième correction du fleuve, entreprise depuis le début des années 2000.

\section{RESTITUER LA GÉOHISTOIRE DU PAYSAGE FLUVIAL}

\section{Méthodologie}

L'approche géohistorique consiste à mettre en interaction constante l'espace et le temps des sociétés (Braudel, 1949; Grataloup, 2015). À travers une perspective diachronique, elle permet d'identifier les trajectoires, les permanences et les inerties d'un espace ou d'un paysage. Il s'agit d'une mise en récits des faits prenant en compte les logiques spatiales et temporelles des sociétés les unes en fonction 

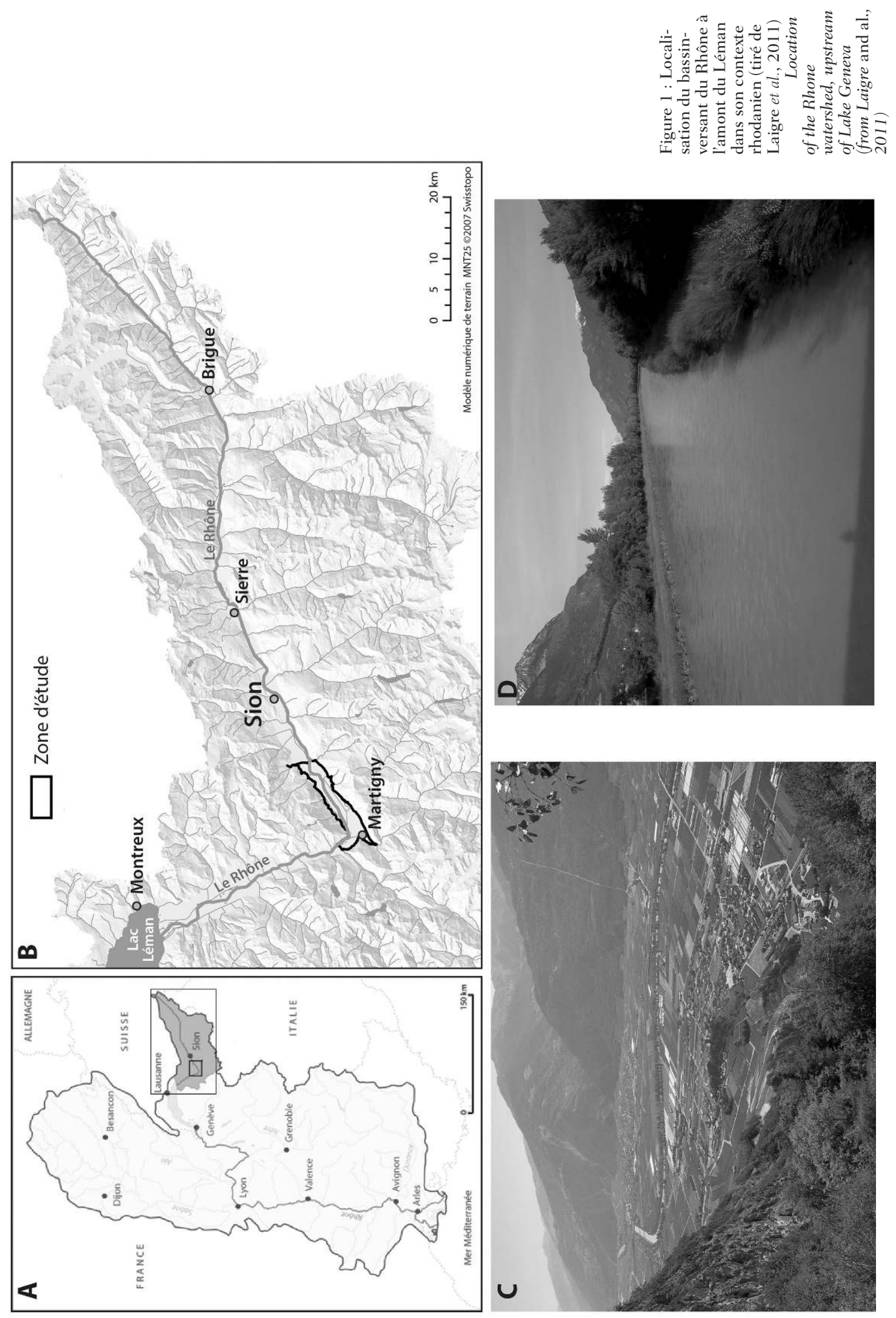

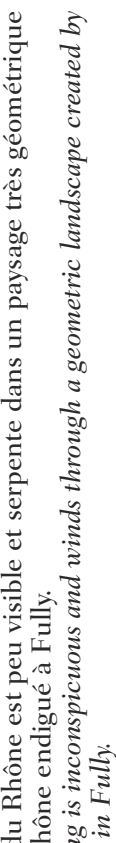



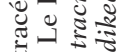

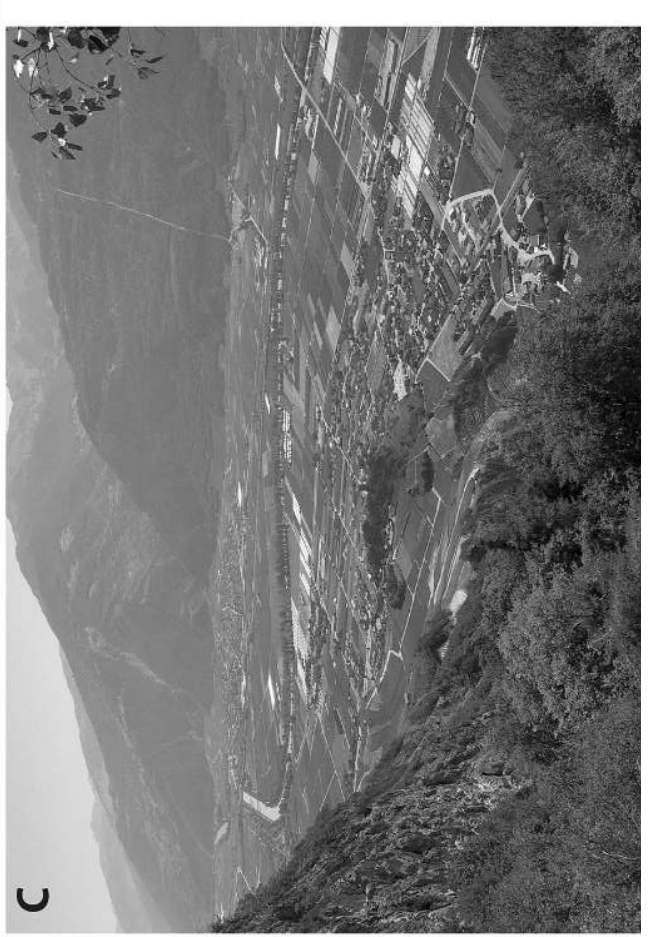

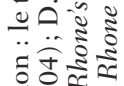



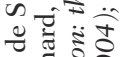

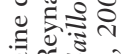



ज.

증

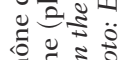

的.

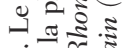

ن



च

ปี



ว

ㄷำ

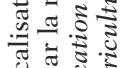

ชี

$\rightarrow$ 
des autres, «le temps devenant aussi un terrain à construire » (Grataloup, 2005). À cet égard, les archives iconographiques et textuelles composent le principal matériau d'une étude géohistorique. Nous avons ainsi constitué pour cette étude un corpus documentaire varié comprenant des ressources planimétriques et plusieurs fonds d'archives. Sont utilisées différentes cartes produites par le gouvernement suisse : les levés originaux et les cartes Dufour (1839 et 1865), l'atlas Siegfried (entre 1879 et 1926$)^{1}$, les cartes nationales de Suisse (dès 1938), ainsi que différents fonds d'archives au sein des Archives de l'État du Valais : les fonds de l'Office des améliorations foncières et du Département des travaux publics du Canton du Valais, un fonds spécifique relatif aux cours d'eau du canton, ainsi que différents fonds de Communes. Des exemplaires des Bulletins des séances du Grand Conseil du Canton du Valais (législatif cantonal), des Protocoles des séances $d u$ Conseil d'État (exécutif cantonal) et des Rapports de gestion du Conseil d'État (rapports annuels sur les activités de l'administration cantonale) ont également été examinés.

Nous avons effectué une cartographie régressive en intégrant les archives planimétriques dans un Système d'Information Géographique (SIG) et en digitalisant l'occupation du sol représentée sur ce corpus. L'objectif était de restituer et analyser les états antérieurs d'utilisation du sol et les formes de terrain ( 5 états de référence déterminés en fonction des sources). Il s'agit d'une démarche couramment utilisée depuis la généralisation des SIG, comme le montrent les travaux de Franchomme et Schmidtt (2012) et de Germaine (2009) à partir du cadastre napoléonien ou encore de Baud (2010) à l'aide du cadastre sarde. Avec cet outil, il est possible de restituer des états de référence au moyen de cartes synchroniques (figures 2 et 6) mais également des évolutions de l'utilisation du sol à l'aide de cartes diachroniques (figures 3, 4 et 5) Ces dernières permettent d'envisager les trajectoires d'occupation du sol (les transitions d'une catégorie d'usage vers une autre) entre les états de référence retenus. Des

1. La carte Dufour au 1:100000 est la première carte topographique officielle de la Suisse couvrant intégralement le pays. Publiée entre 1845 et 1865 , elle est contemporaine de l'émergence de l'État fédéral moderne. Pour cette étude, nous avons utilisé les levés de la carte Dufour au 1:50 000. La carte Siegfried, quant à elle, est un atlas topographique de la Suisse au 1:25000 en plaine et au 1:50000 en montagne. Prenant appui sur deux lois fédérales de 1868, elle fit l'objet d'une coopération entre la Confédération et les cantons. matrices de transition (tableaux 1 et 2) sont créées à partir du croisement des données des cartes synchroniques, livrant ainsi une version quantitative des changements observés. Au préalable, le traitement de ces cartes anciennes implique la prise en compte de leurs caractéristiques techniques afin de corriger les éventuelles distorsions lors de leur intégration dans le $\mathrm{SIG}^{2}$ (par exemple l'ellipsoïde et le système de projection sont différents de ceux qui ont servi à la production des cartes nationales). Le fait que la précision du levé topographique ait évolué entre le XIX siècle et la période actuelle doit également être gardé à l'esprit lors de l'analyse des résultats. Enfin, la représentation des usages du sol sur les cartes anciennes n'est pas constante depuis le $\mathrm{XIX}^{\mathrm{e}}$ siècle. Les vignes ne sont reproduites qu'à partir de l'établissement de l'atlas Siegfried (1880) et les vergers qu'à partir de la réalisation des cartes nationales (1965). Toutefois, d'autres documents d'archives attestent de leur présence dans le paysage depuis au moins le XIX ${ }^{e}$ siècle.

Afin de replacer les différentes trajectoires identifiées dans un contexte plus général et de dépasser l'analyse seule des éléments visibles d'un paysage, les transformations observées doivent être examinées à travers le prisme des événements sociétaux, économiques ou environnementaux en compulsant les fonds d'archives. Les informations contenues dans ces documents permettent de reconstituer les activités et les usages associés aux paysages du passé et d'identifier les ruptures responsables des changements. En complément des traitements cartographiques, l'analyse des archives textuelles autorise la mise en récit des phénomènes observés et dépasse l'inventaire des éléments matériels du paysage, ces derniers pouvant être ainsi reliés aux anciens usages de la plaine alluviale et aux choix opérés par les sociétés contemporaines émanant d'une diversité d'acteurs (les pratiques des riverains du Rhône, les décisions des élus locaux ou régionaux, les mesures législatives cantonales ou nationales liées à l'eau ou à l'agriculture, etc.).

\footnotetext{
2. Pour plus de précisions concernant les cartes utilisées, les problèmes méthodologiques liés aux documents anciens et le traitement des données, se référer aux articles de Reynard (2009) et Baud et al., 2015.
} 


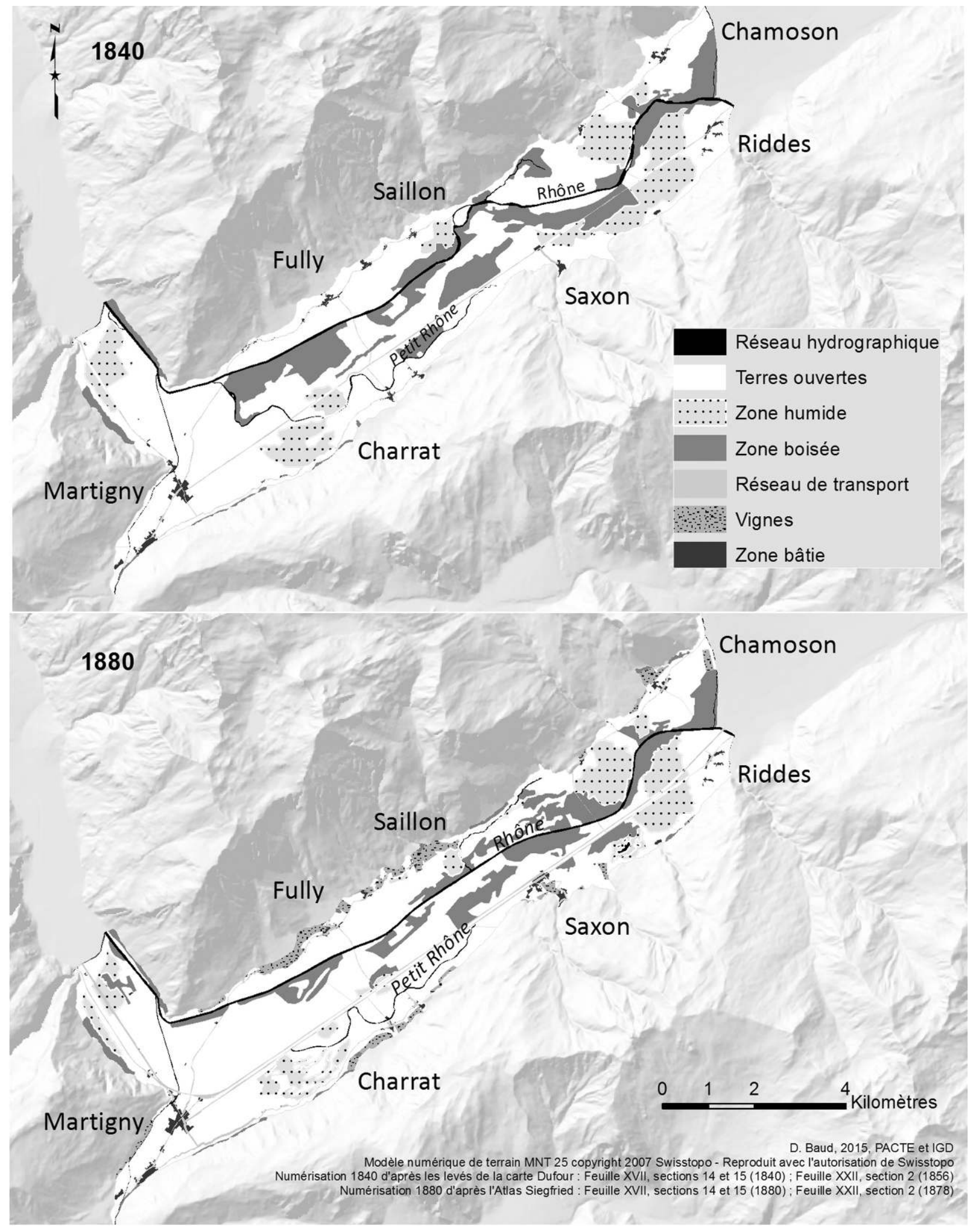

Figure 2 : Cartes synchroniques présentant la répartition de l'utilisation du sol dans la plaine du Rhône entre Riddes et Martigny avant les travaux de la première correction (1840) et quelques années avant la fin des aménagements (1880)

Synchronic maps showing the land use distribution in the Rhône valley between Martigny and Riddes before work of first correction (1840) and a few years before the end of the works (1880) 


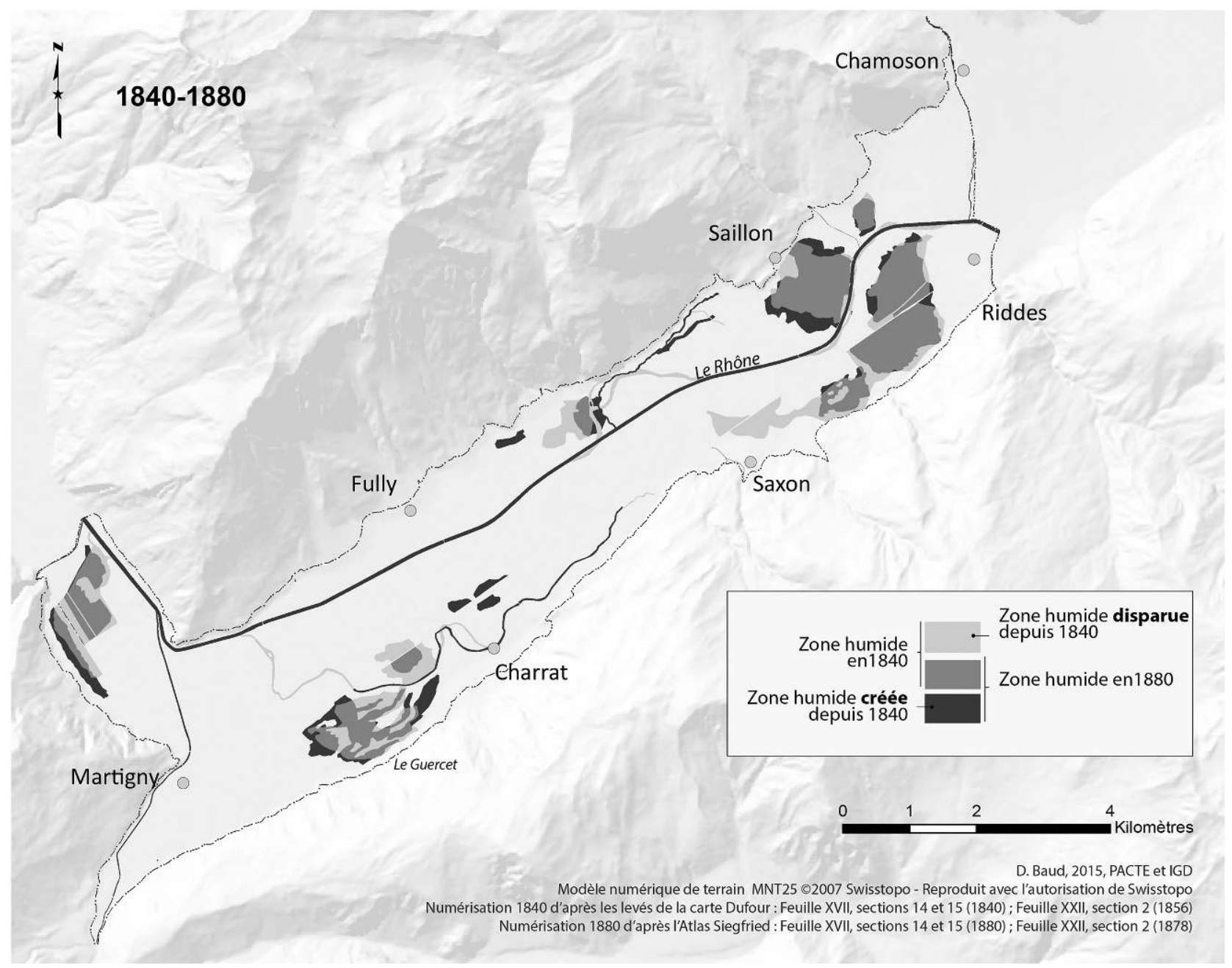

Figure 3 : Carte diachronique présentant l'évolution des zones humides entre 1840 et 1880 Diachronic map showing the evolution of wetlands between 1840 and 1880

\section{Les débuts de la correction systématique du fleuve et les premiers changements paysagers}

Afin de prémunir les sociétés riveraines et leurs activités de l'impétuosité du Rhône et de ses débordements annuels (Pasche, 2004), des aménagements de grande ampleur sont réalisés durant toute la seconde moitié du XIX ${ }^{e}$ siècle, transformant profondément les territoires environnants. Avant cette correction systématique, de petits ouvrages tels que les digues dites « offensives », qui renvoyaient le courant sur la rive opposée du fleuve, étaient construits ponctuellement par les communautés pour protéger des inondations les terrains à vocation agricole, en particulier les pâtures. Toutefois, l'absence de gestion concertée combinée aux faibles moyens dont disposaient les sociétés riveraines entraînaient plutôt des conflits entre les communautés, chacune accusant l'autre «d'expédier le fleuve vers les voisins d'en face » (Scheurer, 2012).

La figure 2 nous livre deux états de la plaine, le premier réalisé à partir de la carte Dufour (1840) avant la correction et le second d'après l'atlas Siegfried (1880) pendant les travaux. Les principales modifications entre les deux dates résident d'une part dans la transformation de la morphologie du fleuve et d'autre part dans les changements et les échanges qui s'opèrent entre les zones boisées et humides et les terres ouvertes. Cette figure témoigne de la première étape du remplacement progressif du paysage fluvial par des terres agricoles amorcé à partir de 1860 . 




Figure 4 : Carte diachronique présentant l'évolution des zones humides entre 1880 et 1920 Diachronic map showing the evolution of wetlands between 1880 and 1920

En 1840, les forêts sont situées essentiellement le long du Rhône, sur une ancienne bande de tressage (Saillon-Fully). La présence de zones boisées à proximité immédiate du cours d'eau, dans un secteur tressé en cours d'abandon, semble indiquer que le fleuve a suivi un tracé similaire pendant plusieurs années, pour que la végétation ait pu ainsi coloniser de vastes étendues. Il s'agit certainement d'un effet de la période d'accalmie relative qui a débuté peu après des débordements catastrophiques survenus en 1782 et qui a duré jusqu'à la crue de 1834 (Scheurer, 2012). Les zones humides sont localisées pour la plupart dans des bas-fonds isolés par les cônes de déjection et les bandes de tressage du Rhône (entre Riddes et Saxon, par exemple). La vaste zone humide de Charrat peut également être expliquée par le barrage du cône du torrent de la Dranse (Martigny) et de l'ancienne bande de tressage du Rhône (Fully).

Sur la carte de 1880, nous distinguons clairement le nouveau tracé du Rhône endigué et redressé de celui de l'ancien cours divaguant entre les cônes de déjection des torrents latéraux où sont établies les zones d'habitations. Le lit a été fortement contraint par un système combinant des digues longitudinales insubmersibles et des épis transversaux redirigeant l'eau vers la partie centrale du fleuve afin de protéger les digues et d'accentuer le flux des sédiments. Il en résulte un profil double avec un lit majeur artificiel (correspondant plus ou moins à l'extension des épis) entourant un lit mineur dans lequel les eaux du fleuve sont concentrées (De Torrenté, 1964). Cette 


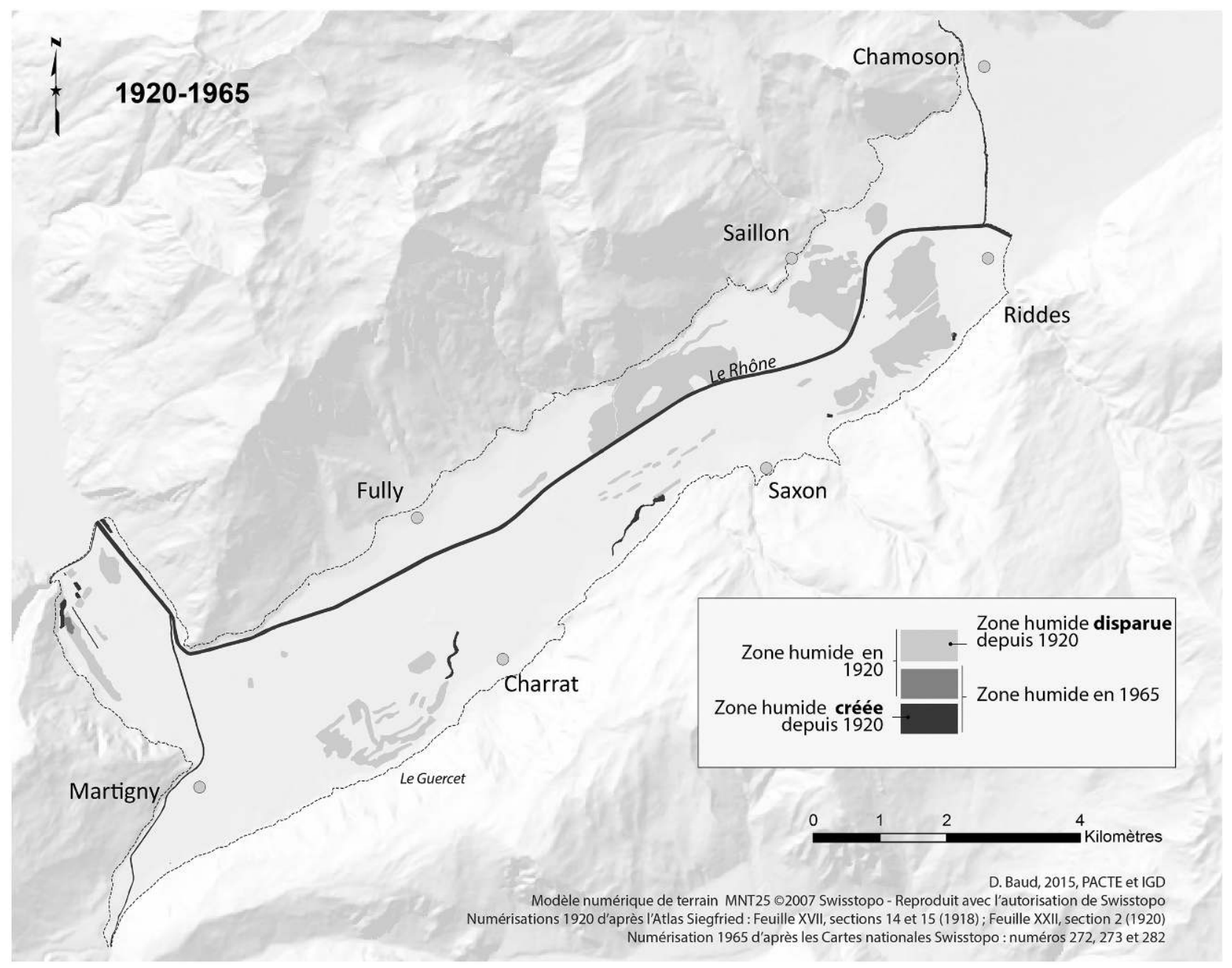

Figure 5 : Carte diachronique présentant l'évolution des zones humides entre 1920 et 1965 Diachronic map showing the evolution of wetlands between 1920 and 1965

première correction a également redressé le tracé du fleuve, s'employant à le rendre plus droit et régulier. En ce sens, quelques méandres ont été coupés aux endroits les plus sinueux afin d'augmenter la pente et donc la vitesse, permettant un transport alluvial plus abondant (secteur de Saillon). Ce nouveau tracé est resté identique jusqu’à aujourd'hui.

Certains chenaux, témoins d'une bande de tressage active durant le Petit Âge Glaciaire (Laigre et al., 2012), se sont déconnectés du Rhône et ont disparu. Le bras secondaire du Petit Rhône, quant à lui, reste encore visible malgré un assèchement progressif entre Charrat et son embouchure dans le fleuve.

Concernant les forêts alluviales, leur emprise au sol a diminué sur l'ensemble du territoire étudié, notamment à proximité du cours d'eau. Afin de connaître quel type d'occupation du sol a remplacé les zones boisées, nous avons généré une matrice de transition (tableau 1) en croisant les données contenues dans les deux cartes. Cette matrice informe des changements d'utilisation du sol intervenus entre les deux époques, ainsi que des espaces qui sont restés stables. La diagonale en grisé désigne ces surfaces qui subsistent dans le même usage du sol. La lecture de gauche à droite permet de repérer les transformations opérées entre 1840 et 1880 pour chaque type d'occupation. Ainsi, 429 hectares de forêt sont restés stables. En revanche, les zones boisées perdent $17 \%$ de leurs surfaces, dont 339 hectares au profit des terres ouvertes. L'expression « terres ouvertes » regroupe les prés et les cultures représentés de manière indifférenciée sur les cartes anciennes. Cette progression des terres ouvertes au 


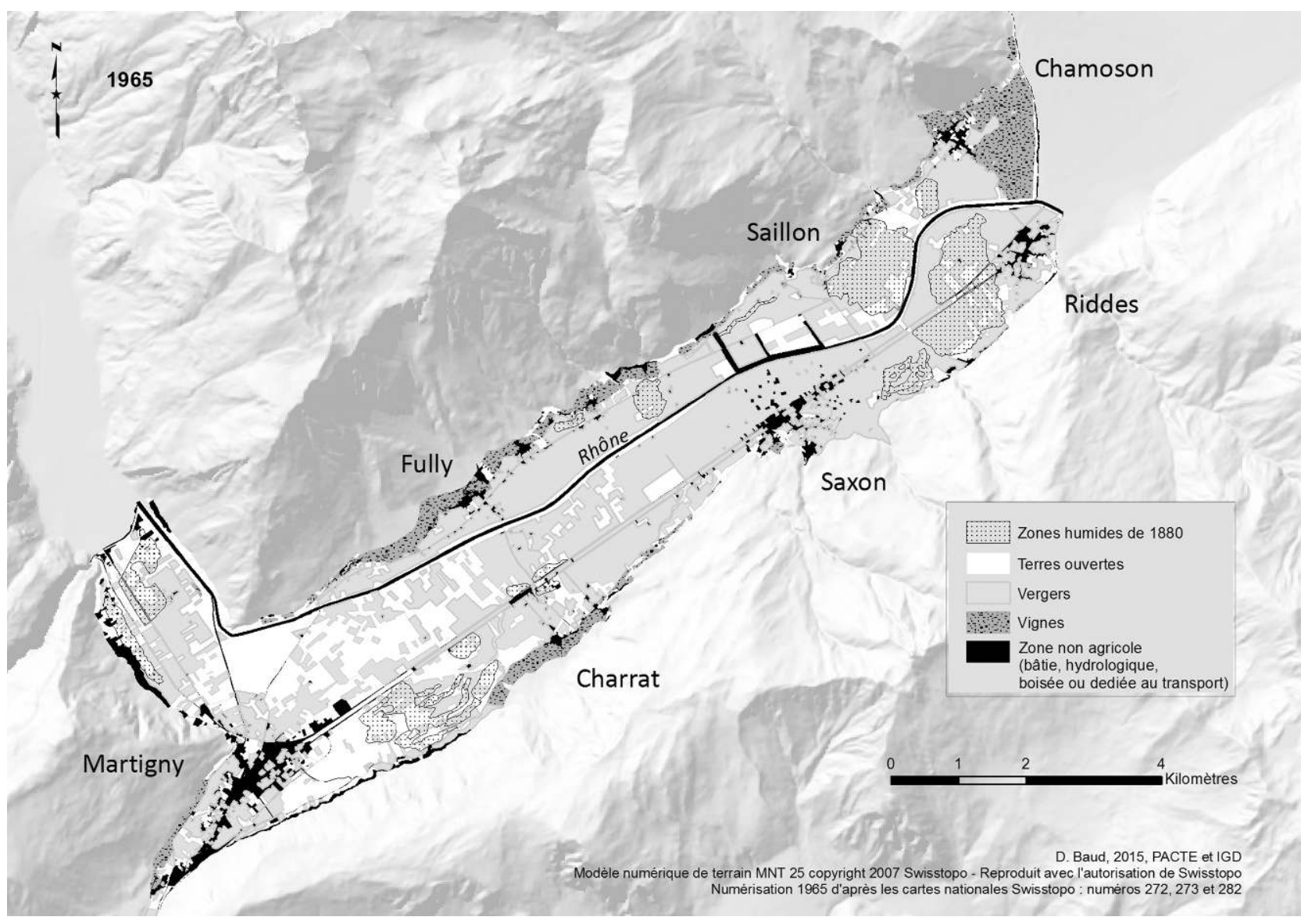

Figure 6 : Carte synchronique présentant l'occupation du sol en 1965 suite aux travaux de corrections et de mise en valeur de la plaine Synchronic map showing land use in 1965 following the work of corrections and development of the plain

détriment de la forêt alluviale correspond au commencement de la mise en valeur systématique de la plaine permise par l'endiguement récent du Rhône. Toutefois, sur cette même période, certains secteurs forestiers se sont plutôt étoffés, comme à l'amont de Saillon, principalement au détriment des terres ouvertes (202 ha). La matrice permet d'observer des échanges de surfaces entre les forêts alluviales et les prés et les champs à un moment où la plaine vit au rythme des travaux agricoles et où le paysage entre en transition. Au final, environ un cinquième des zones boisées est ainsi récupéré par les riverains pour l'exploitation de nouvelles cultures ou la création de prairies.

Enfin, les marais qui occupent une place prépondérante dans la plaine, au point d'inciter certains voyageurs du XIX ${ }^{\mathrm{e}}$ siècle à comparer ce territoire rhodanien à la Camargue provençale en regard du climat et de l'humidité (Bender, 1996), connaissent également ce jeu d'échanges avec les terres ouvertes. La première correction n'est que l'étape initiale des aménagements qui plongent ces territoires humides au cœur d'enjeux environnementaux et sociétaux jusqu'au milieu du $\mathrm{Xx}^{\mathrm{e}}$ siècle. Les marais vont faire l'objet de trajectoires variées, et leurs dynamiques à l'origine du modelage progressif du paysage fluvial sont abordées précisément dans la deuxième partie.

\section{L'ÉMERGENCE D'UNE NOUVELLE ORGANISATION DES FORMES PAYSAGÈRES}

Entre la fin du XIX ${ }^{e}$ siècle et le début du XX $x^{e}$ siècle, l'aménagement du cours d'eau modifie le paysage fluvial en permettant une première mise en valeur de nouveaux territoires sensés être désormais sécurisés. Toutefois, comme nous allons le voir ci-après, le bilan de la première correction, plutôt mitigé, 


\begin{tabular}{|c|c|c|c|c|c|c|c|c|c|}
\hline & & \multicolumn{7}{|c|}{1880} & \multirow[b]{2}{*}{ Total 1840} \\
\hline & Surface (ha) & Hydro & $\begin{array}{c}\text { Terres } \\
\text { ouvertes }\end{array}$ & $\begin{array}{l}\text { Zones } \\
\text { boisées }\end{array}$ & $\begin{array}{l}\text { Zones } \\
\text { bâties }\end{array}$ & $\begin{array}{c}\text { Zones } \\
\text { humides }\end{array}$ & $\begin{array}{l}\text { Réseau } \\
\text { transport }\end{array}$ & Vignes & \\
\hline \multirow{9}{*}{1840} & Hydro & 103 & 34 & 14 & 0 & 4 & 1 & 1 & 156 \\
\hline & Terres ouvertes & 10 & 2314 & 202 & 17 & 108 & 36 & 155 & 2842 \\
\hline & Zones boisées & 16 & 339 & 429 & 0 & 16 & 4 & 3 & 807 \\
\hline & Zones bâties & 0 & 0 & 0 & 65 & 0 & 1 & 0 & 67 \\
\hline & Zones humides & 5 & 190 & 20 & 1 & 406 & 6 & 1 & 629 \\
\hline & Réseau transport & 0 & 1 & 0 & 0 & 0 & 49 & 1 & 51 \\
\hline & Total 1880 & 134 & 2878 & 666 & 83 & 534 & 97 & 161 & \\
\hline & Changement (ha) & -22 & 30 & -141 & 17 & -95 & 46 & - & \\
\hline & Changement (\%) & -16 & 1 & -17 & 20 & -15 & 47 & & \\
\hline
\end{tabular}

Tableau 1 : Matrice de transition montrant les trajectoires d'occupation du sol entre 1840 et 1880 Transition matrix showing the trajectories of land use between 1840 and 1880



Tableau 2 : Matrice de transition montrant les trajectoires d'occupation du sol entre 1880 et 1920 Transition matrix showing the trajectories of land use between 1880 and 1920

annonce déjà les prémisses d'une seconde phase de travaux. Il s'agit d'une époque charnière où les Valaisans réorganisent leur territoire à travers des choix d'activités économiques et environnementaux qui détermineront en grande partie l'occupation de l'espace et les modes de gestion des ressources à l'origine des paysages fluviaux actuels, l'objectif étant de gagner un maximum de terres cultivables sur les territoires occupés par le Rhône.

Suite à la première correction du fleuve, les riverains et les ingénieurs chargés du projet vont rapidement constater une augmentation de l'humidité dans les régions basses de la plaine, principalement entre les cônes de déjection des affluents du Rhône. La carte diachronique de 1840 à 1880 (figure 3) témoigne de la création de ces surfaces marécageuses notamment aux marges des zones humides préexistantes (aux environs de Saillon, Riddes et Charrat). Les travaux d'endiguement n'avaient certes pas vocation à drainer les marais; toutefois, l'apparition de cette dynamique imprévue par le projet va bouleverser pendant plusieurs dizaines d'années les activités agro-pastorales dans les secteurs touchés. Dès 1867, la presse locale relaye le mécontentement des riverains : «On peut prévoir que l'endiguement du Rhône ne produira point les fruits qu'on en avait fait espérer. Déjà sur plusieurs points, on a observé l'augmentation des marais par suite de la filtration et du défaut d'écoulement des eaux de source » (Le Confédéré, 4 août 1867). De 
nombreux témoignages d'experts du Département des Ponts et Chaussés ou de la Commission rhodanique - chargée de la surveillance des travaux d'endiguement et d'assainissement de la plaine attestent, dans les rapports ou les courriers, de la diminution des cultures au profit du développement des marais entre la fin du XIX ${ }^{\mathrm{e}}$ siècle et le début du $\mathrm{Xx}^{\mathrm{e}}$ siècle $^{3}$ (108 ha de terres ouvertes deviennent des zones humides d'après le tableau 1). Cette augmentation des surfaces humides est liée au fait que les eaux de ruissellement et des cours d'eau secondaires sont piégées entre les digues et les points hauts que forment les cônes de déjection. Comme la nappe phréatique est elle-même haute en raison des barrières naturelles formées par les cônes de déjection, ces eaux de ruissellement peinent à s'infiltrer et forment des mares temporaires - notamment à la fonte des neiges - et des marais plus ou moins permanents. La situation ne s'améliorera que lorsque les embouchures des affluents dans le Rhône seront systématiquement aménagées et que de grands travaux de drainage seront entrepris.

Dans le secteur du «Guercet », ce ne sont pas les travaux sur le Rhône qui entraînent les transformations mais ceux de la construction de la ligne de chemin de fer du Simplon, inaugurée en 1860, et qui, sur les points les plus bas de la plaine, a été construite sur des remblais importants afin de sécuriser la voie des inondations. Ces remblais ont formé une digue qui a joué un rôle déterminant dans ce milieu humide en créant un espace hydrologique fermé. Les eaux de ruissellement se sont ainsi retrouvées bloquées et ont continué de stagner. De plus, lors de la construction des remblais, des matériaux ont été prélevés de part et d'autre dans la plaine, sur une largeur de trente mètres de chaque côté des voies (Pasche, 2004), entraînant un phénomène de creusement des fonds alentours. La combinaison de ces deux facteurs - l'établissement de remblais formant une digue et le creusement des secteurs à proximité de la voie - a entretenu et même accentué l'expansion de la zone humide, ce qui révèle une méconnaissance, de la part des constructeurs de la ligne du Simplon, de la micro-

3. AEV Fonds Cours d'eau, 6300 - 2, réf. 281, Rapport sur l'assainissement de la plaine Sion-Riddes, de MM. Stockalper, Gaillard et Udry, août 1893. AEV Fonds Cours d'eau, 6300 - 2/316, Lettre du Conseil d'Etat au Conseil fédéral du $1^{\text {er }}$ août 1911 concernant une demande de subvention fédérale pour l'assainissement de la plaine de Riddes au Trient. topographie de la plaine et des héritages morphologiques du Rhône.

Afin de contrecarrer ce processus et de gagner un maximum de terrains cultivables sur les zones de marais, le canton du Valais a engagé deux opérations d'aménagement : d'une part, l'endiguement de l'embouchure des affluents durant les trois dernières décennies du XIX ${ }^{\mathrm{e}}$ siècle, et d'autre part, l'élaboration par le Département des travaux publics d'un projet général d'assainissement en 1873. Ce dernier prévoyait le creusement d'un collecteur principal et d'une série de canaux secondaires sensés empêcher les infiltrations du fleuve en direction de la plaine. Entre Riddes et Martigny, un premier canal est aménagé suite à l'adoption du plan d'assainissement, produisant assez peu d'améliorations dans un premier temps, comme le montre la carte diachronique de 1880 à 1920 (figure 4) qui indique toujours une forte empreinte des zones humides dans le paysage, seule une infime partie de leur surface ayant diminué sur l'ensemble de la zone étudiée ( -7 ha ; tableau 2). Il faudra attendre l'approfondissement du collecteur principal et le déplacement de son embouchure vers l'aval, à proximité du Trient, ainsi que la réalisation de plusieurs canaux secondaires, pour que la situation s'améliore.

D'après la matrice de transition, des échanges se poursuivent entre les terres ouvertes, les forêts alluviales et les zones marécageuse (par exemple 40 ha de marais sont devenus des bois et une surface pratiquement identique de forêt, 48 ha, s'est transformée en zone humide). Un nouveau marais s'est même créé en rive droite du Rhône entre Saillon et Fully sur des terres cultivées, suite à la construction d'une digue visant à se protéger des inondations d'un affluent : la Sarvaz. En complément des trajectoires croisées du triptyque marais/forêts alluviales/terres agricoles, la vigne renforce sa présence dans le paysage valaisan en doublant quasiment sa superficie au détriment des terres ouvertes (144 ha) et des zones boisées ( 31 ha). Les sociétés locales misent sur cette activité depuis plusieurs dizaines d'années en la développant sur les cônes de déjection.

Entre 1860 et 1920, l'endiguement du Rhône et de ses affluents visant à sécuriser la plaine amorce le façonnement d'un paysage fluvial géométrique tout en déclenchant des bouleversements dans l'organisation de l'espace et des activités humaines. Outre les modifications majeures de la morphologie flu- 
viale, nous constatons l'émergence de trajectoires paysagères non linéaires car les diminutions ou les progressions des surfaces de zones humides, boisées et agricoles ne sont pas homogènes à l'échelle du territoire d'un point de vue spatio-temporel. Des marais apparaissent sur des terres anciennement cultivées ou pâturées, provoquant le mécontentement des riverains mais surtout une nécessité d'intervention afin de redéfinir les enjeux économiques de la plaine. Après avoir dompté le Rhône, les autorités vont chercher à maîtriser les dynamiques de reconquête des marais et des zones boisées dans le but de cultiver ou de planter des arbres fruitiers sur l'ensemble du territoire. Suite au bilan mitigé des travaux de drainage lancés en 1873, les élus et décideurs cantonaux se tournent alors vers la Confédération afin d'obtenir une aide pour la mise en valeur des terrains. En 1914, une subvention fédérale destinée à l'assainissement de la plaine de Martigny est octroyée et avec elle s'enclenche une réelle dynamique de mise en valeur agricole qui se prolongera jusqu'à la fin des années quarante (Baud et al., 2015).

\section{LA REDÉFINITION DES ENJEUX ENVIRONNEMENTAUX ET SOCIO- ÉCONOMIQUES DE LA PLAINE DU RHône}

Dans la continuité de la première correction du fleuve, l'assèchement systématique de la plaine s'étale sur plus de 60 ans, jusqu'à la fin des années 1930 et participe activement à la transition paysagère de la plaine. Sa mise en œuvre est émaillée par des décisions politiques majeures (principalement les décisions de subventionnement cantonal et fédéral de 1873 et 1914), divers épisodes de crues (notamment en 1868, 1896, 1897, 1902 et 1920), qui mettent à mal les résultats de certains travaux, ainsi que de nombreux conflits et tensions entre acteurs impliqués dans cette vaste entreprise (Baud et al. 2015). C'est pourquoi on remarque un décalage, d'un point de vue temporel, entre les aménagements du fleuve et les changements paysagers majeurs entrâ̂nés par la mise en valeur agricole de la plaine.

\section{Une première période de litiges}

Le projet d'assainissement de 1914, subventionné par la Confédération, comprend l'approfondis- sement des canaux créés à la fin du XIX ${ }^{\mathrm{e}}$ siècle et la densification du réseau de canaux secondaires. Cette deuxième phase a également pour objectif de pallier aux problèmes de refoulement et de remontée de la nappe. Sur le secteur d'étude, les travaux se déroulent entre 1918 et 1934 et sont à l'origine de conflits entre communes mais également avec d'autres acteurs comme les Chemins de fer fédéraux (CFF). En effet, chaque communauté a son propre rythme dans la réalisation des travaux en fonction de ses finances et d'autres projets prioritaires (eau potable et routes, notamment $)^{4}$, ce qui ne convient pas toujours aux territoires les plus avancés, qui préféreraient terminer les opérations d'assainissement rapidement. Ensuite, les CFF s'opposent à certains aménagements, craignant que de nouveaux travaux ne viennent déstabiliser la ligne de chemin de fer et n'entraînent un tassement de la voie ${ }^{5}$. La Commission rhodanique, chargée de statuer sur le problème, ne partagera pas cet avis et recommandera le maintien de la participation financière des CFF, nécessaire à l'avancement de l'assèchement.

Pendant les premières années de mise en route du programme d'assainissement, les litiges sont nombreux, notamment dans la répartition financière entre les différents acteurs. Toutefois, au fur et à mesure que les résultats sont décrits comme concluants, les conflits deviennent plus marginaux, surtout à partir de la fin des années 1920.

\section{Vers de nouveaux modes de gestion et d'aménagement des espaces ruraux}

Au final, les travaux d'assainissement vont prolonger le collecteur principal vers l'aval ${ }^{6}$ et permettre un approfondissement du canal et un drainage plus profond des terrains, entraînant l'assèchement des anciens marais, comme nous le montre la figure 5. En effet, la quasi-totalité des zones marécageuses disparaît entre 1920 et 1965 . De petits îlots humides apparaissent ponctuellement (par exemple

4. AEV Fonds Cours d'eau, $6300-2 / 281$.

5. AEV Fonds Cours d'eau, 6300 - 2, réf. 281, Rapport complémentaire de la Commission rhodanique concernant les propositions de répartition des travaux d'assainissement de la Plaine entre Sion et Riddes, 5 novembre 1920.

6. Au lieu de rejoindre le Rhône au niveau du coude qu'il forme entre Fully et Martigny, le canal se jette maintenant dans le Rhône au niveau du Trient, à l'extrémité occidentale de la zone étudiée. Ce prolongement du collecteur permet d'abaisser le point d'embouchure et donc d'abaisser le profil général du canal. 
aux gouilles de Guercet) mais cela reste très anecdotique.

Les travaux directement liés à l'assèchement systématique du territoire ne sont pas les seuls à l'origine de la disparition des zones humides. En complément, les sociétés riveraines ont opéré d'autres types d'aménagement afin de développer économiquement leur plaine et de s'orienter vers une culture intensive. La mise en valeur a été progressive et rendue possible à l'aide de remaniements parcellaires, du développement de l'irrigation, de défrichements, du nivellement de la plaine, ou encore de l'arasement des dunes éoliennes.

Dans les années 1920, plusieurs communes, comme Saillon et Riddes, réorganisent leur territoire en effectuant des remembrements parcellaires. Dans un même temps, des réseaux d'irrigation sont également réalisés sur des secteurs devenus trop secs pour l'agriculture suite à l'enfoncement des canaux et collecteurs. Cette pratique se généralisera par la suite à l'échelle de toute la plaine afin de favoriser un meilleur rendement agricole. Au début des années 1940, de nombreux rapports du Service cantonal des améliorations foncières font état de la part de surface cultivée aux abords des villages, avec par exemple une estimation de 95 à $98 \%$ pour la plaine de Martigny-Ville ou encore $90 \%$ pour celle de Fully $^{7}$. Ces bons scores dont se réjouissent les ingénieurs sont complétés par des descriptions paysagères mentionnant « de beaux vergers et de grandes étendues en cultures d'asperges et de fraises ${ }^{8} »$. Le bilan des opérations d'assainissement, d'irrigation ou encore de remaniement parcellaire est très positif et annonce déjà l'enjeu suivant : mettre en culture intensive tous les terrains encore en friche de la plaine. Cet objectif intervient dans un contexte de deuxième guerre mondiale et de hausse démographique significative où il devient nécessaire de cultiver la plus petite parcelle de terrain, (par exemple à Fully, la population est passée de 1800 à 2700 habitants en vingt ans $\left.{ }^{9}\right)$. Pour inciter les propriétaires riverains à défricher les dernières terres incultes, le canton prévoit de verser des subsides à ceux

7. AEV, 3320-3 Améliorations foncières, 141-1072/1, Martigny-Ville, Mémoire de l'Ingénieur rural, 1944. L'estimation est calculée en fonction de la surface totale de la commune.

8. AEV, 3320-3 Améliorations foncières, 135-1012/1, Fully, Mémoire concernant le projet d'extension des cultures, 1942.

9. AEV, 3320-3 Améliorations foncières, 135-1002/1, Fully, Rapport de contrôle, 1941. qui fourniront la preuve des travaux. En quelques années, les terrains vagues restants sont déboisés et nivelés, permettant la plantation d'arbres fruitiers ou le maraîchage. Les dernières dunes existantes (dont certaines pouvant mesurer jusqu'à 3 mètres de haut), issues de vieux bras du Rhône ou de processus éoliens, sont également nivelées.

L'assainissement systématique de la plaine et ses opérations connexes (remaniements parcellaires, défrichements, irrigation, nivellement du sol et arasement des dunes) ont entraîné de profondes transformations du paysage, avec le remplacement entre 1920 et 1965 des zones humides par des espaces agricoles (figure 6). Les terres ouvertes et les vergers, qui sont enfin représentés sur les sources cartographiques, sont les principaux gagnants des nouveaux terrains asséchés. L'activité fruitière implantée depuis les années 1870 (Loup, 1965) connaît un véritable essor, une accélération, en dépassant la simple production locale, suite aux travaux « d'améliorations » de la plaine. Le vignoble a également profité des aménagements en développant sa surface sur le bas des cônes de déjection, comme à Chamoson par exemple (Reynard et al., 2007).

Cette période marque un véritable tournant dans l'exploitation de la plaine, avec la disparition quasitotale des zones marécageuses au profit du développement intensif des surfaces arboricoles et marâ̂chères. La réorganisation de la plaine se termine et marque la fin de la transition de ce paysage fluvial. Afin d'avoir une vue synthétique de la localisation des transformations majeures effectuées sur le secteur entre Riddes et Martigny, la figure 7 présente trois exemples de trajectoires d'occupation du sol entre 1840 et 1965 représentatives des changements observés et décrits tout au long de la présente étude. Le tableau en haut à gauche indique la temporalité de ces trois trajectoires et leur parcours au sein des grandes catégories d'occupation du sol (nous avons regroupé sous le vocable de «zones agricoles » les terres ouvertes, les vignes et les prés ainsi que le bâti et le réseau de transport sous celui de «zones artificialisées »). Dans la partie droite de la figure, la carte schématise les zones dont les changements résultent d'une de ces trois trajectoires.

Si comme nous l'avons vu précédemment, les transformations ne sont pas linéaires dans le temps, elles ne sont pas non plus homogènes à l'échelle du 

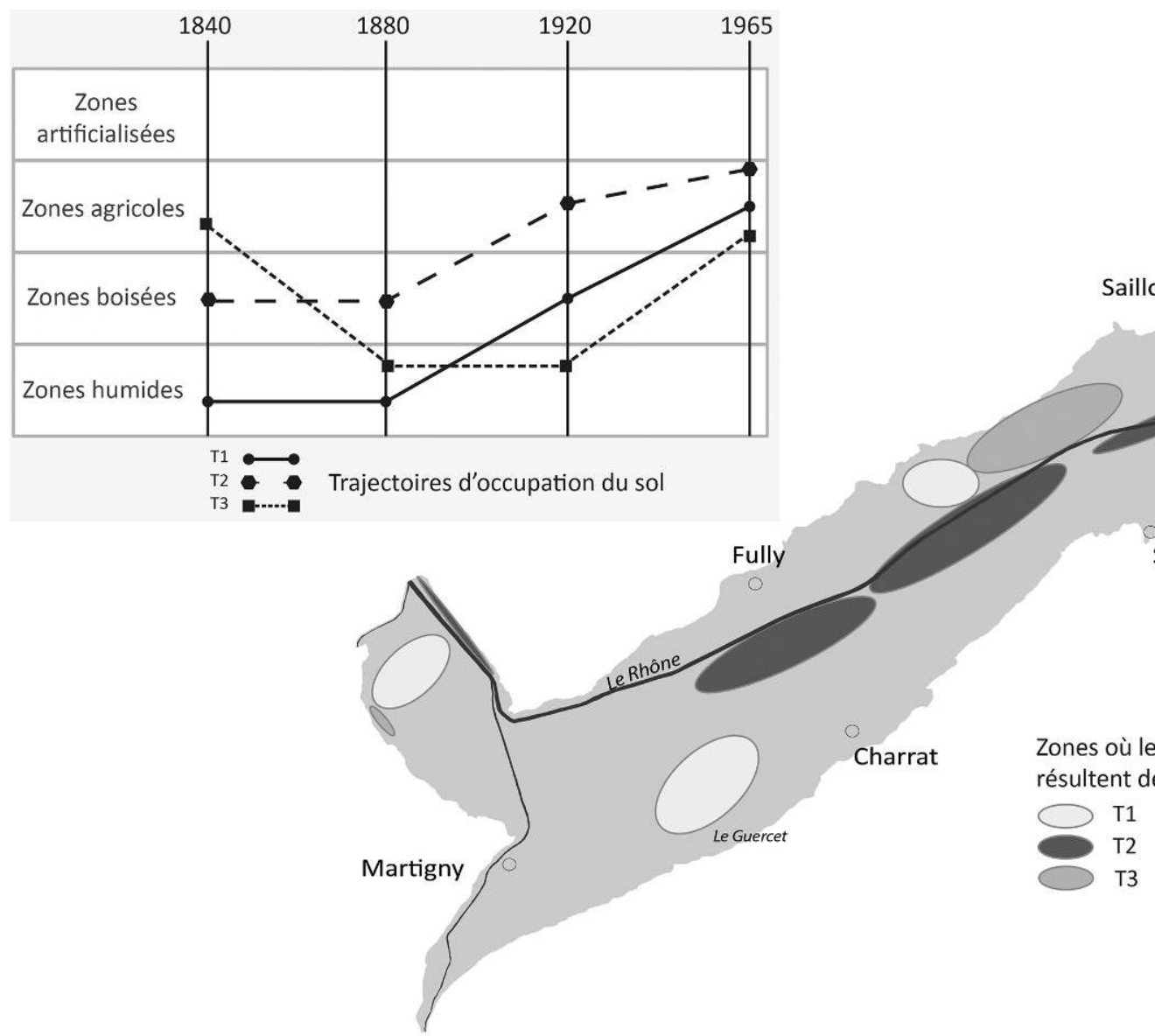

Saxon

D. Baud, 2015, PACTE et IGD

Figure 7 : Les transformations majeures du territoire à partir de trois trajectoires représentatives. The most important transformations territory from three representative trajectories.

territoire. Par exemple la trajectoire T2 ne concerne que les espaces situés à proximité immédiate du Rhône. Ces secteurs, initialement boisés en 1840 et issus souvent d'anciens bancs de galets et graviers, ont été difficiles à défricher, ce qui explique leur stabilité dans ce mode d'occupation du sol avant d'être mis en valeur (cultures puis vergers). Les zones affiliées à la trajectoire $\mathrm{T} 1$ sont quant à elles plus dispersées sur l'ensemble du territoire étudié. Il s'agit des espaces humides les plus persistants qui ont été tardivement valorisés. Enfin, la trajectoire T3 concerne des zones jouxtant les secteurs de trajectoires Tl car il s'agit des espaces de terres ouvertes qui ont été humidifiées suite aux dynamiques non prévues des travaux d'aménagement.

À l'aide de cette démarche diachronique et à partir de la typologie d'occupation du sol, quelques grandes tendances de trajectoire peuvent ainsi être identifiées (figure 8) :
- les trajectoires stables, lorsque la zone reste identique à sa catégorie initiale : espaces agricoles (prés, cultures, vergers ou vignes), surfaces boisées, zones humides ou espace artificialisé (bâti, réseau de transport);

- les trajectoires de transformation faible, lorsque les changements s'opèrent à l'intérieur d'une même catégorie (par exemple une terre ouverte devient un verger);

- les trajectoires de bifurcation, où l'on observe le passage d'un mode d'occupation du sol à un autre (par exemple une zone humide est mise en culture).

Les trois exemples de trajectoires paysagères (figure 7) combinent ces grandes tendances montrant que le paysage de la plaine alluviale a été sans cesse en mouvement, se recomposant tout au long de sa transition au rythme des opérations d'aménagement et des décisions des pouvoirs locaux et cantonaux. 
Figure 8 : Schéma des grandes tendances de trajectoires paysagères.

Diagram of the major trends of landscape trajectories.



Trajectoire stable
Trajectoire de faible transformation
Trajectoire de bifurcation

\section{Conclusion}

Au cours des 150 dernières années, la physionomie de la plaine du Rhône valaisan a radicalement changé. Avec le déclenchement des travaux de la première correction du fleuve, le paysage est entré dans une phase de transition conduisant à un autre système d'organisation socio-spatial. Au milieu du $\mathrm{XIX}^{\mathrm{e}}$ siècle, les riverains s'adaptent au fleuve et à son environnement de zones humides et boisées, en « mettant en valeur » les terrains cultivables et les pâtures au gré des crues et des changements de tracé dans un système agro-pastoral plutôt basé sur l'autosubsistance.

Un siècle plus tard, le fleuve au tracé originellement sinueux, est géométriquement contraint par les différentes opérations de correction visant à sécuriser le territoire contre les crues. Un paysage composé d'un éventail d'activités agricoles, s'est peu à peu développé en lieu et place des anciennes forêts alluviales et des marais suite à l'assèchement progressif de ces terrains permettant des cultures intensives. Plus tard, dès les années 1960, une partie de ces terres agricoles seront elles-mêmes réduites par l'étalement urbain à proximité des villages historiques et par la construction d'infrastructures de transport (routes, autoroute) (Stäuble et Reynard 2005; Baud et al., 2015).

L'approche géohistorique à l'aide de cartes anciennes permet de caractériser et de localiser finement les transformations qui ont participé à cette transition paysagère. Leur analyse met en évidence des trajectoires d'occupation du sol et des permanences qui ne sont ni linéaires dans le temps ni homogènes dans l'espace. En complément, les archives textuelles informent sur les processus et les décisions prises par les acteurs à l'origine de ces changements.

Différentes dynamiques de projets d'aménagement ont été abordées dans cet article. Ce sont tout d'abord des changements effectués sur le fleuve lui-même, avec des travaux qui le canalisent, le rectifient, le relèguent à une ligne dans le paysage, avec en parallèle une première «mise en valeur » de terrains engagée par les sociétés riveraines. Puis, rapidement, l'augmentation des zones humides mise en évidence par la carte diachronique de la figure 3 vient perturber ce processus d'amélioration de la plaine. L'endiguement entraîne des effets non désirables et les propriétaires doivent s'adapter à cette contrainte non sans signifier leur mécontentement concernant l'humidification non prévue de leurs terrains. Il s'agit d'une période d'inertie où espaces boisés et humides progressent dans certains secteurs pendant que les riverains et les décideurs locaux cherchent des solutions et des financements (la première phase d'assainissement n'aura pas l'effet escompté). C'est ensuite l'organisation de l'assèchement pendant sa réalisation qui a une incidence directe sur le paysage. Le choix des techniques, la création des canaux et l'avancement au gré des financements de façon inégale dans la plaine entraînent une discontinuité paysagère entre les terrains mis en valeur et les îlots en attente de déboisement ou d'assèchement. Et parfois, certaines évolutions sont contrées par les décisions ou les choix techniques; c'est le cas des terrains déjà mis en valeur du point de vue agricole dans la plaine de Martigny qui voient leur qualité se péjorer (assèchement et nécessité de recourir à l'irrigation) durant 
la deuxième phase d'assainissement dans les années 1920 (Baud et al., 2015). Enfin, l'assainissement est vecteur de débats au sein des différentes communautés suite à la submersion de certains terrains ou à l'abaissement de la nappe à d'autres endroits. Ainsi, les grandes évolutions de la plaine du Rhône ont profondément marqué le paysage mais également les riverains qui ont été associés aux processus d'aménagement et de mise en valeur de leur espace.

Les transformations des paysages fluviaux du Rhône entre la fin du XIX ${ }^{e}$ siècle et aujourd'hui sont le résultat d'une double dynamique d'aménagement du cours d'eau (première correction) assez précoce et, surtout, d'une mise en valeur agricole après 1920. Les modifications paysagères majeures de la plaine consistent au remplacement des paysages fluviaux (dès 1860) et des paysages de zones humides (plutôt après 1920), par des terres cultivées. L'intensification agricole a entraîné la réduction des paysages ouverts herbagers et des paysages de milieux humides avec un certain décalage d'un point de vue temporel en regard des interventions sur le fleuve lui-même, un décalage qui avait déjà été mis en évidence plus à l'amont, dans la région de Conthey (Stäuble et Reynard, 2005). Au final, c'est bien la combinaison - se succédant dans le temps - d'une politique de protection contre les crues, initiée à partir des années 1860, comme ailleurs en Suisse d'ailleurs (Vischer, 2003), et d'une politique de mise en valeur agricole des terres jusque-là incultes, surtout après la première guerre mondiale, qui créée le paysage géométrique de l'agriculture intensive, décrit par Jean Loup (1965).

Aujourd'hui, les travaux de la Troisième correction ont débuté sur le secteur de Viège. Le projet, dont les principes ont été adoptés en 2000 par le Grand Conseil (législatif cantonal), vise à protéger les biens et les personnes en intégrant les intérêts environnementaux et socio-économiques. En raison de visions contrastées concernant le poids respectif des aspects sécuritaires, environnementaux et économiques, le projet est toutefois marqué par de vives controverses entre acteurs aux intérêts différents. En remontant dans le temps pour mettre en lumière les dynamiques territoriales et sociétales qui ont marqué les travaux d'aménagement dans le passé, l'étude géohistorique du fleuve et de sa plaine prend ici tout son sens.

\section{Bibliographie}

Baud D., 2010. Étude des dynamiques d'occupation du sol et des paysages alpins: des archives cadastrales des Pays de Savoie aux Systèmes d'Information Géographique, Thèse de doctorat de géographie, Université de Savoie, 352 p.

Baud D., Reynard E., Bussard J., 2015. Les transformations paysagères de la plaine du Rhône. Analyse diachronique et cartographie historique (1840-2010), in Reynard E. (dir.), Le Rhône entre nature et culture, Sion, coll. « Cahiers de Vallesia; $29 »$.

Bender G., 1996. De la Camargue à la Californie. La plaine, le Rhône et les riverains, enjeux, débats et réalisations dans la région de Martigny, 1750-1860, Mémoire de licence, Université de Genève, n.p.

Braudel F., 1949. La Méditerranée et le monde méditerranéen à l'époque de Philippe II, Paris, Armand Colin; deuxième édition révisée, 1966, 2 volumes, 589 p. et 629 p.

Clément V., 1999. Pays et paysages forestiers de Vieille Castille ( $\mathrm{xI}^{\mathrm{e}}-\mathrm{XX}^{\mathrm{e}}$ siècle), Annales de Géographie, tome 108, n 609 610, p. 651-655.

De Torrenté C., 1964. La correction du Rhône en amont du lac Léman, Berne, Département fédéral de l’intérieur, n.p.

Franchomme M., Schmidtt G., 2012. Les zones humides dans le Nord vues à travers le cadastre napoléonien : les Systèmes d'Information Géographique comme outils d'analyse, Revue du Nord, tome 94, p. 661-680.

Germaine M.-A., 2009. De la caractérisation à la gestion des paysages ordinaires des vallées dans le Nord-Ouest de la France. Représentations, enjeux d'environnement et politiques publiques en BasseNormandie. Thèse de doctorat de géographie, Université de Caen Basse-Normandie, 648 p.

Grataloup C., 2005. Géographie historique et analyse spatiale : de l'ignorance à la fertilisation croisée, in Boulanger P., Тrochet J.-R. (dir.), Oì en est la géographie historique? Entre économie et culture, Paris, p. 33-42.

Grataloup C., 2015, Introduction à la géohistoire, Paris, Armand Colin, coll. «Cursus », 224 p.

Jaсов-Rousseau N., 2009, Géohistoire/Géo-histoire. Quelles méthodes pour quel récit?, Géocarrefour, n 84, p. 211-216.

Laigre L., Reynard E., Arnaud-Fasseta G., Baron L., Glenz D., 2012. Caractérisation de la paléodynamique du Rhône en Valais central (Suisse) à l'aide de la tomographie de résistivité électrique, Géomorphologie : relief, processus, environnement, $\mathrm{n}^{\circ} 4$, p. 405-426.

Loup J., 1965. Pasteurs et agriculteurs valaisans. Contribution à l'étude des problèmes montagnards, Grenoble, Allier, 427 p.

Pasche L., 2004. Travaux de correction des cours d'eau en Valais et dans la région de Conthey (1860-1900), Vallesia, tome 59, p. 225-246.

Périgord M., Donadieu P., Barraud R., 2012. Le paysage, Armand Colin, Paris, $2^{\mathrm{e}}$ édition, 126 p.

Reynard E., 2009. Les sources cartographiques pour l'histoire du Rhône valaisan, in Reynard E., Evéquoz-Dayen M., Dubuis P. (dir.), 2009. Le Rhône: dynamique, histoire et société, Sion, «Cahiers de Vallesia; 21 », 238 p. 
Reynard E., Evéquoz-Dayen M., Borel G. (dir.), 2009. Le Rhône, entre nature et société, Sion, Cahiers de Vallesia 21, $342 \mathrm{p}$.

Scheurer A., 2012. Le Rhône et ses riverains entre Riddes et Martigny (1400-1860). Quatre longs siècles de conflits et de solutions. IV. Quarante ans de projets, de travaux, de litiges et de catastrophes (1820-1860), Vallesia, vol. 67, p. 1-67.

Stäuble S., Reynard E., 2005. Évolution du paysage de la plaine du Rhône dans la région de Conthey depuis 1850.
Les apports de l'analyse de cartes historiques, Vallesia, vol. 60, p. 433-456.

Vischer D. L., 2003. Histoire de la protection contre les crues en Suisse - Des origines jusqu'au 19e siècle, Berne, Office fédéral des eaux et de la géologie, 208 p.

Zanini F., Zanini E., Weber C., Schlaepfer R., 2006. Analyse de la dynamique du paysage de la plaine du Rhône de 1850 à 2003 sur la base de cartes topographiques, Bull. de la Murithienne, tome124, p. 89-98. 